\title{
Downregulation of cathepsin L suppresses cancer invasion and migration by inhibiting transforming growth factor- $\beta$-mediated epithelial-mesenchymal transition
}

\author{
QINGQING ZHANG $^{1 *}$, MEILING HAN $^{1 *}$, WENJUAN WANG $^{1}$, YUNZHEN SONG $^{1}$, \\ GANG CHEN $^{2}$, ZHONG WANG ${ }^{2}$ and ZHONGQIN LIANG ${ }^{1}$ \\ ${ }^{1}$ Department of Pharmacology, College of Pharmaceutical Sciences, Soochow University; \\ ${ }^{2}$ Department of Neurosurgery, The First Affiliated Hospital of Soochow University, Suzhou, Jiangsu, P.R. China
}

Received September 22, 2014; Accepted December 22, 2014

DOI: $10.3892 / o r .2015 .3754$

\begin{abstract}
Cathepsin L, a lysosomal acid cysteine protease, was found to be overexpressed in several types of human carcinomas. However, its functional roles in tumor progression and the underlying mechanisms remain largely unclear. In the present study, we investigated a novel functional aspect of cathepsin $\mathrm{L}$ in regulating transforming growth factor- $\beta$ (TGF- $\beta$ )-induced epithelial-mesenchymal transition (EMT) in A549 and MCF-7 cells and examined its possible mechanisms. We found that TGF- $\beta$-induced cell morphologic changes of EMT were associated with the increased protein level of cathepsin L in A549 and MCF-7 cells, suggesting that cathepsin L may be involved in the regulation of EMT. Furthermore, we showed that silencing of cathepsin L blocked TGF- $\beta$-induced cell migration, invasion and actin remodeling and inhibited TGF- $\beta$-mediated EMT. We also demonstrated that the mechanism of how cathepsin L knockdown regulates EMT may be explained by the suppression of EMT-inducing molecules, such as Snail, which is associated with the phosphatidylinositol 3-kinase (PI3K)-AKT and Wnt signaling pathways. Moreover, we proved that cathepsin L knockdown in A549 cells significantly inhibited xenograft tumor growth and EMT in vivo. The results showed a new mechanism to determine cathepsin $\mathrm{L}$ involvement in the regulation of cancer invasion and migration. These results showed that cathepsin $\mathrm{L}$ knockdown is important in regulating EMT and suggest that cathepsin L may be utilized as a new target for enhancing the efficacy of chemotherapeutics against epithelial cancer.
\end{abstract}

Correspondence to: Dr Zhongqin Liang, Department of Pharmacology, College of Pharmaceutical Sciences, Soochow University, 199 Renai Road, Suzhou, Jiangsu, P.R. China

E-mail: liangzhongqin@suda.edu.cn

*Contributed equally

Key words: cathepsin L, EMT, invasion, migration, epithelial cancer

\section{Introduction}

The epithelial-mesenchymal transition (EMT) program is the differentiation switch epithelial polarized cells into motile mesenchymal cells, which plays a key role during various biological events such as embryonic development, fibrotic diseases and tumor metastasis. EMT enables cells to acquire fibroblast-like properties and shows reduced intercellular adhesion and increased motility (1-5). Furthermore, EMT is dysregulated in cancer cells and is characterized by the acquisition of mesenchymal phenotype, leading to enhanced motility and allow tumor cells to metastasize and establish secondary tumors at distant sites $(3,6)$. Additionally, EMT contributes to enhanced chemoresistance and the acquisition of stem/progenitor-like characteristics by regulating signaling pathways (7). Findings of those studies suggest that EMT is involved in the modulation of drug resistance and enhances the ability of tumor metastasis.

Transforming growth factor- $\beta$ (TGF- $\beta$ ), a secreted cytokine, was found to act as a suppressor in tumor cell growth control, however, it may also promote tumor invasion and metastasis (8). Accumulating evidence reveals that TGF- $\beta$ is often used as the inducer of EMT $(9,10)$ to increase tumor metastasis. Although several molecules involved in TGF- $\beta$-mediated EMT have been identified, the mechanisms involved in the induction of EMT remain to be determined.

Cathepsin $\mathrm{L}$ is a cysteine protease that belongs to the papain-like family (peptidase $\mathrm{C} 1 \mathrm{~A}$ ), which is reported to be associated with cancer tumorigenesis, proliferation and migration (11-14). It plays an important role in the degradation and renewal of intracellular proteins, and is involved in several important physiological processes including the activation of prohormone, presenting antigens and development of organs (15-17). Cathepsin L has been proven to be upregulated in a variety of malignancies: breast, lung, gastric, colon, melanomas and gliomas. Moreover, the level of cathepsin L expression is associated with the degree of malignancy (18). Results of previous studies showed that cathepsin $\mathrm{L}$ inhibition increases apoptosis via the responsiveness of IGF-1 receptor (19), or the activity of arsenite in U87 cells (20). Simultaneously, in cathepsin L antisense clones of 
U87 cells, the apoptotic rate is increased when induced by intrinsic or extrinsic stimulation (21). Furthermore, accumulating evidence suggests cathepsin $\mathrm{L}$ has been associated with tumor invasion and migration $(22,23)$. When the extracellular activity of cathepsin $\mathrm{L}$ is increased, the cell-cell adhesion is reduced and degradation of the ECM is increased (24,25-27). In addition, overexpression of cathepsin L was contributed to the increased malignant properties following the Ras-transformation of NIH/3T3 cells (28). Frade et al, Yang et al and Rousselet et al proved that the upregulation of cathepsin L also may switch the melanoma cell phenotype from non-metastatic to highly metastatic and increased tumor invasion and migration (29-31). The aforementioned studies showed that cathepsin $\mathrm{L}$ increased the invasion and migration of ovarian cancer cells and may therefore be a molecular target for cancer metastasis (32).

Cathepsin L is known to regulate tumor progression, invasion and migration. However, the mechanisms involved in the regulation of tumor invasion and migration remain to be determined. Findings of previous studies showed that cathepsin $\mathrm{L}$ may contribute its proteolytic action to the migration and invasion of tumor cells (24-27). Increased activity of cathepsin $\mathrm{L}$ decreased the cell-cell adhesion and enhanced degradation of the ECM, suggesting that downregulation of cathepsin L decreased metastatic tumor development (33). However, Goulet et al suggested that the role of cathepsin L in cancer metastasis may be associated with its extracellular activities and may involve its processing function of the transcription factor CUTL1 (34). Other authors reported that cathepsin L is also involved in proteolytic activation cascades, which include u-PA and cathepsin B. Of note, this proteolytic network plays an important role in tumor invasive processes (35). On the other hand, it has been reported that inhibition of cathepsin L did not affect invasion of 8863 and LM melanoma cell lines (36). Nevertheless, the exact role of cathepsin $\mathrm{L}$ in tumor cell invasion and migration remains unidentified, and many studies suggest that it is context-dependent.

On the basis of the crucial role of cathepsin $\mathrm{L}$ and its involvement in tumor invasion and migration, we investigated the effect of cathepsin L knockdown on tumor invasion and migration and whether cathepsin L enhanced tumor via invasion and migration TGF- $\beta$-mediated EMT. We demonstrated that cathepsin $\mathrm{L}$ is a regulator of tumor invasion and migration in cancer cells, and the regulation of tumor invasion and migration by cathepsin L is mediated through EMT. Thus, cathepsin $\mathrm{L}$ is a novel target for reducing tumor progression.

\section{Materials and methods}

Materials. Cell culture reagents and the Lipofectamine reagent were purchased from Invitrogen Life Technologies (Carlsbad, CA, USA). Human recombinant TGF- $\beta$ was purchased from R\&D Systems (Minneapolis, MN, USA). Phalloidin was obtained from Sigma-Aldrich (St. Louis, MO, USA). The antibodies used in this study were: N-cadherin (Abcam, Cambridge, MA, USA); E-cadherin (BD Biosciences, Franklin Lakes, NJ, USA); cathepsin L (Abcam); Akt and p-Akt (both from Cell Signaling Technology, Danvers, MA, USA); Snail and Wnt-5a (both from Santa Cruz Biotechnology, Inc., Santa Cruz, CA, USA); $\beta$-actin (MultiSciences Biotech Hangzhou,
China); and Cxcr-7 (Anbo Biotechnology, Sunnyvale, CA, USA).

Cell culture. A549 and MCF-7 cells and their derivative lines were cultured in RPMI-1640 supplemented with $10 \%$ fetal bovine serum (FBS), penicillin $(100 \mathrm{U} / \mathrm{ml}) /$ streptomycin $(100 \mathrm{U} / \mathrm{ml})$, and 300 or $500 \mathrm{ng} / \mathrm{ml}$ of puromycin at $37^{\circ} \mathrm{C}$ in a humidified atmosphere of $5 \% \mathrm{CO}_{2}$. All the knockdown and control sublines from these cell lines were generated using cathepsin L shRNA plasmid and control vectors shRNA plasmid (both from Santa Cruz Biotechnology, Inc.) under puromycin selection.

Wound-healing and invasion assays. For the wound-healing assay the cells were grown in 6-well plates. When confluency was achieved, the cells were scratched by a pipette tip, rinsed to remove debris, then further incubated with fresh RPMI-1640 containing 1\% FCS in the presence or absence of TGF- $\beta$ for $24 \mathrm{~h}$. Cell migration images were captured at 0 and $24 \mathrm{~h}$, quantitative analysis of the wound-healing index determined as a percentage was calculated using 20 randomly selected distances across the wound at 0 and $24 \mathrm{~h}$, divided by the distance measured at $0 \mathrm{~h}$.

A cell invasion assay was performed using 24-well Matrigel invasion chambers (BD Biosciences). The cells were trypsinized and reseeded in the upper chamber at a concentration of $1 \times 10^{5} / \mathrm{ml}$ in $200 \mu \mathrm{l}$ of RPMI-1640 supplemented with $1 \%$ FCS. The lower chamber contained $700 \mu 1$ of RPMI-1640 supplemented with $10 \%$ FCS. After $24 \mathrm{~h}$, the cells on the upper surface of the filters were removed, and the cells on the lower surface were fixed with methanol and stained with crystal violet.

Determination of Snail mRNA levels by reverse transcription-PCR. Cells were grown in 6-well plates, and treated or not with TGF- $\beta$ for $24 \mathrm{~h}$. Total RNA was isolated with TRIzol reagent according to the manufacturer's instructions. RNA was reverse transcribed and amplified by PCR using the primers: Cathepsin L upstream: 5'-AAACACAGCTTCACA ATGGCC-3' and downstream: 5'-TTTGAAAGCCATTCA TCACCTG-3'; Snail upstream: 5'-CCTCCCTGTCAGATG AGGAC-3' and downstream: 5'-CCAGGCTGAGGTATTCC TTG-3'. The amplification products were analyzed in a $1.0 \%$ agarose gel.

Immunofluorescence. Twenty-four hours after seeding on coverslips with TGF- $\beta$ the cells were fixed with methanol for $10 \mathrm{~min}$ at $4^{\circ} \mathrm{C}$ and permeabilized for $10 \mathrm{~min}$ with $0.1 \%$ Triton $\mathrm{X}-100$. The cells were then incubated for $1 \mathrm{~h}$ in blocking buffer (1\% BSA and $0.1 \%$ Triton $\mathrm{X}-100)$ at $4^{\circ} \mathrm{C}$. For immunofluorescence (IF), the cells were incubated with antibodies against E- and $\mathrm{N}$-cadherin at $4^{\circ} \mathrm{C}$ overnight. The cells were then rinsed three times with $\mathrm{PBS}$, and incubated with appropriate biotinylated secondary antibodies for $1 \mathrm{~h}$. Alex Fluor 488 (green) (1:500; Molecular Probes, Eugene, OR, USA) was used as a third antibody for $1 \mathrm{~h}$. Nuclei were counterstained with $0.5 \mathrm{ng} / \mathrm{ml}$ DAPI for $15 \mathrm{~min}$ at room temperature. Coverslips were mounted on slides with vectashield mounting medium for fluorescence and analyzed by confocal microscopy.

Actin cytoskeleton staining. Cells were fixed for $10 \mathrm{~min}$ in $4 \%$ paraformaldehyde, permeabilized with $0.2 \%$ Triton 
A

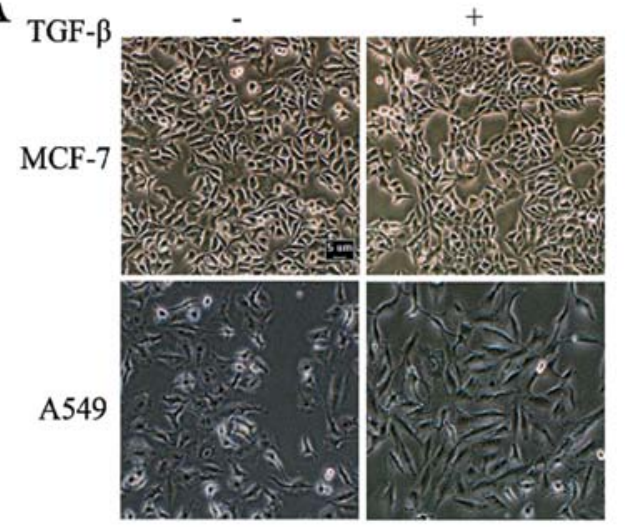

B
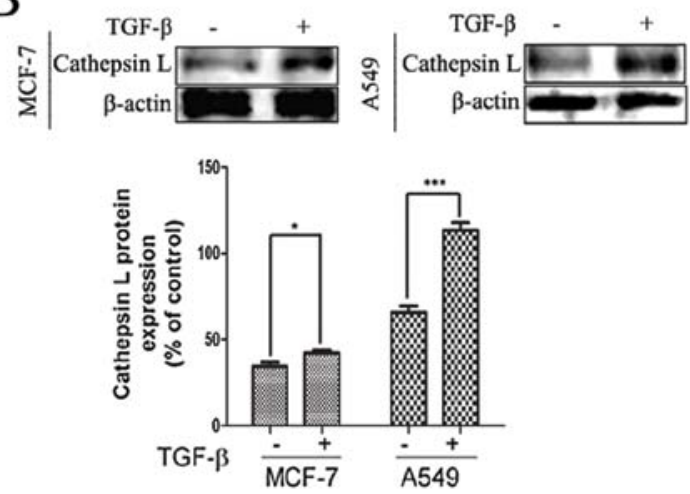

Figure 1. The effect of TGF- $\beta$ on cell morphology and cathepsin L expression in cells. A549 and MCF-7 cells were treated with TGF- $\beta$ ( 5 and 10 ng/ml) for 24 h. (A) Cell morphology was observed using microscopy. Images were captured using a 10x objective lens. (B) Expression level of cathepsin L was examined by western blot analysis. At least three independent experiments were performed. ${ }^{*} \mathrm{P}<0.05,{ }^{* *} \mathrm{P}<0.01$ and ${ }^{* * *} \mathrm{P}<0.001$ compared with control. TGF- $\beta$, transforming growth factor- $\beta$.

$\mathrm{X}-100$ in PBS for $10 \mathrm{~min}$, blocked in $2 \% \mathrm{BSA}$ for $30 \mathrm{~min}$, and incubated for $1 \mathrm{~h}$ with phalloidin. The nuclei were counterstained with DAPI.

Western blot analysis. The samples were harvested and lysed with ice-cold lysis buffer. The protein concentration of the lysates was determined by Protein Assay Kit (Thermo Fisher Scientific, Rockford, IL, USA). The lysates were loaded and separated on $10 \%$ SDS-PAGE gels and the proteins were transferred onto a polyvinylidene difluoride membrane. The membrane was blocked in 5\% BSA for $1 \mathrm{~h}$, and incubated with primary antibodies overnight. After washing three times, the blots were incubated with secondary antibodies for $1 \mathrm{~h}$. Immunoblots were detected by the Odyssey Infrared Imaging System (Li-COR Biosciences, Lincoln, NE, USA).

In vivo assay and immunohistochemical staining. Five-week-old male nude (BALB/c) mice (Animal experiment Center of Suzhou University, Suzhou) wsere used in the present study A549-shVector and A549-shCathepsin L cells (5x10 ${ }^{7}$ cells $/ 0.1 \mathrm{ml}$ of medium/mouse) were injected into mice subcutaneously to generate the mouse model. On day 42 after tumor injection, the tumors were removed from mice and analyzed for western blotting and immunohistochemical staining. The samples were stained using VECTASTAIN ABC kit and ImmPACT DAB (Vector Laboratories, Burlingame, CA, USA) according to the supplier's instructions. Tumor size was measured with a caliper and was calculated using the formula: $\pi / 6$ x larger diameter $\mathrm{x}$ (smaller diameter $)^{2}$.

\section{Results}

TGF- $\beta$ induces morphological changes and enhances the expression of cathepsin $L$ in cancer cells. To determine the effect of TGF- $\beta$ on cell morphology and the expression level of cathepsin L, we observed the morphological changes and examined the activity of invasion and migration in A549 human lung and in human MCF-7 breast cancer cell lines treated with TGF- $\beta$ for $24 \mathrm{~h}$. As shown in Fig. 1A, treatment of the human A549 and MCF-7 cancer cell lines with
TGF- $\beta$ revealed morphological changes, with a more scattered and spindle-shaped appearance, especially in A549 cells (Fig. 1A). TGF- $\beta$ also significantly increased the expression of cathepsin L, which has been reported to play an important role in cancer invasion and migration (Fig. 1B). Furthermore, TGF- $\beta$ induces EMT characteristics such as loss of cell-cell adhesion, reorganization of actin cytoskeleton and acquisition of increased migratory characteristics and promotion of cell migration $(3,37)$. On the basis of previous exploration, an association was identified between cathepsin L and the EMT induced by TGF- $\beta$. Thus, we determined whether cathepsin L is a target of TGF- $\beta$ in regulating EMT, which regulates tumor invasion and migration.

Cathepsin L knockdown attenuates TGF- $\beta$-induced cell migration and invasion. The regulation of cathepsin $\mathrm{L}$ by TGF- $\beta$ suggests that cathepsin L is involved in TGF- $\beta$-induced cell migration and invasion. To verify this role of cathepsin $\mathrm{L}$, shRNA expression vectors were introduced into A549 and MCF-7 cells, and stably transfected cell clones were obtained by limiting dilution culture under the pressure of puromycin. RT-PCR and western blot analysis were conducted to reveal loss of cathepsin L expression in RNA and protein levels in the cathepsin L knockdown (shcathepsin L) cells (Fig. 2A). In tumor cell lines treated with TGF- $\beta$, respectively, the effect of cathepsin L knockdown on migration and invasion was determined by a wound-healing assay. The results showed an increase in the width of the injured line. Results of the colony formation assay exhibited a decrease in the number of colonies, suggesting that suppression of cathepsin L inhibits TGF- $\beta$-induced cell migration and invasion in A549 and MCF-7 cells (Fig. 2B and C). The effect of cathepsin L was more significant in A549 than in MCF-7 cells. Furthermore, we demonstrated that suppression of cathepsin L markedly suppressed TGF- $\beta$-induced actin remodeling associated with cell motility in A549 and MCF-7 cells (Fig. 2D). In addition, emerging evidence suggests that multiple pairs of chemokines and their receptors are likely to play important roles in mediating tumor growth and metastasis (38). TGF- $\beta$ treatment enhanced the expression of $\mathrm{Cxcr}-7$, one of the chemokine 
A
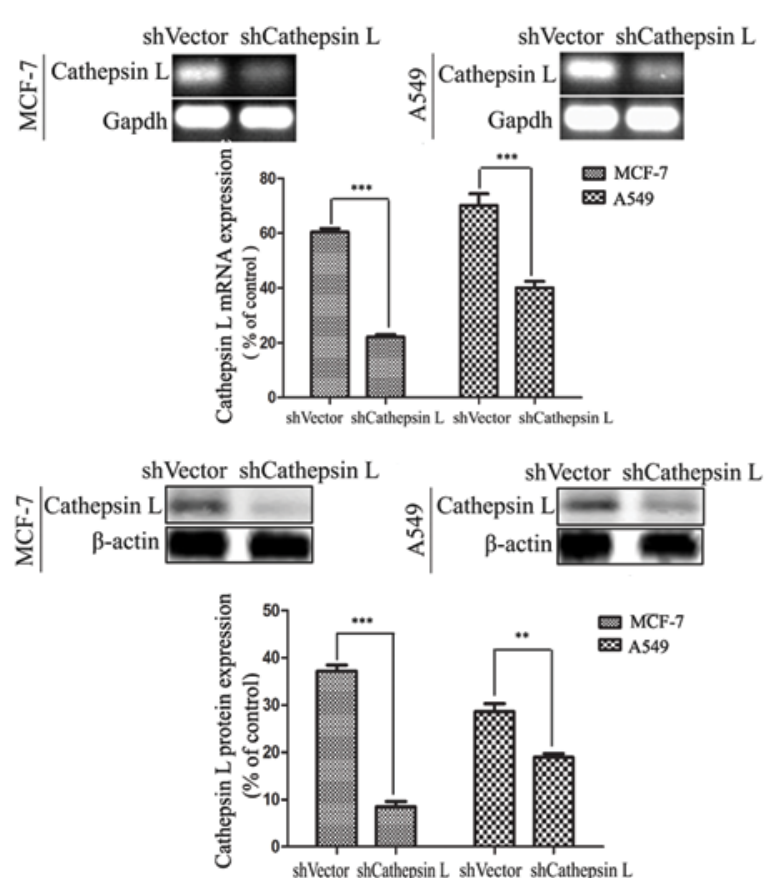

C
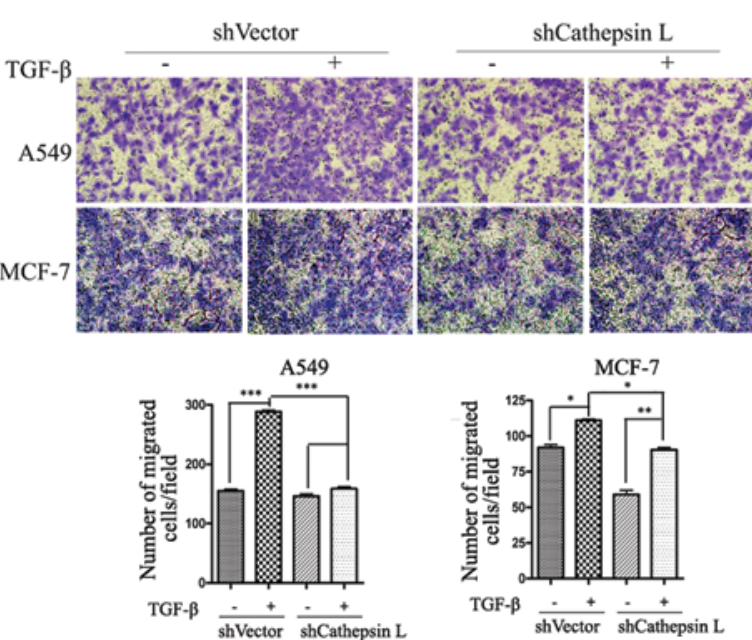

B

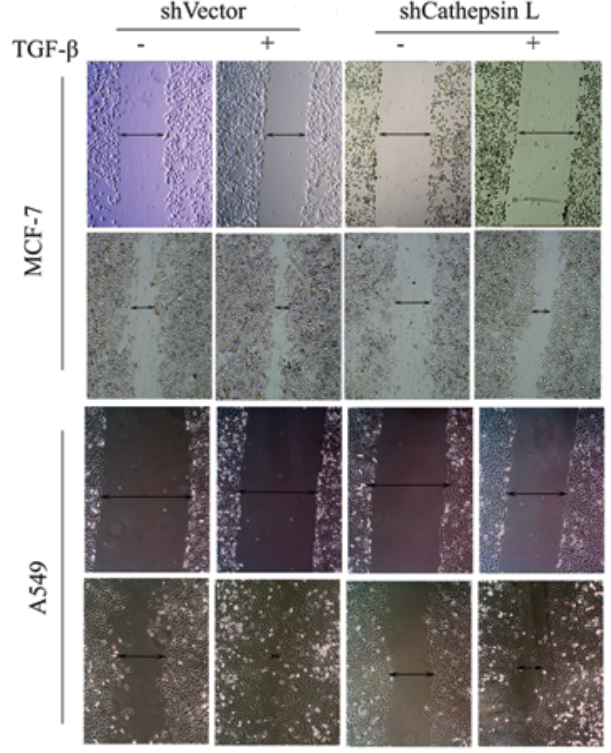

$\mathrm{D}$

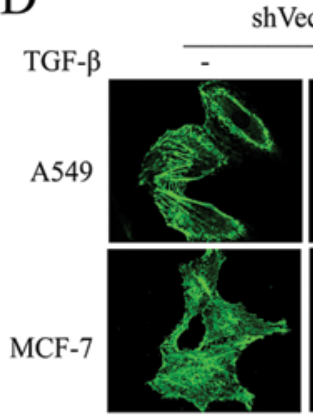

shVector
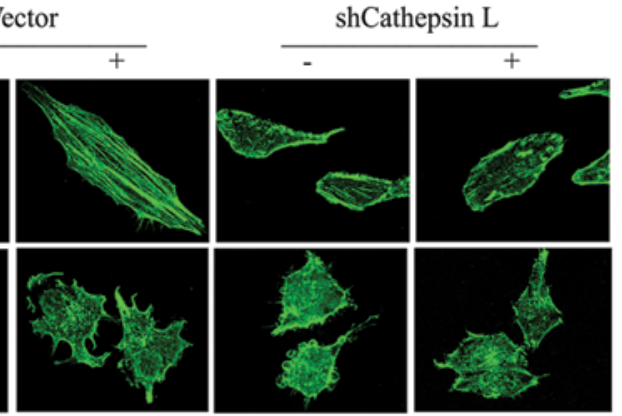

E
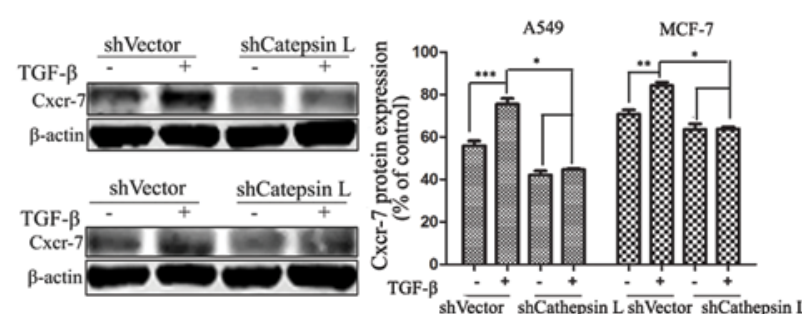

Figure 2. Stable transfection of shCathepsin L blocks TGF- $\beta$-induced cell migration and invasion. (A) Western blot analysis was performed to determine the expression level of cathepsin L of A549 and MCF-7 cells transfected with cathepsin L shRNAs targeting the human cathepsin L sequence or the control shRNA (shVector). (B) Effect of cathepsin L inhibition on TGF- $\beta$-induced cell migration. Images were captured using a 4x objective lens. (C) Effect of cathepsin L inhibition on TGF- $\beta$-induced cell invasion. Images were captured using a 20x objective lens. (D) Representative phalloidin staining for actin-cytoskeleton in A549 and MCF-7 cells. Spreading of cells was analyzed after plating on collagen-coated slides. At the indicated time points, the cells were fixed, stained with phalloidin, and visualized by fluorescent microscopy. Images were captured using a 40x objective lens. (E) Effect of cathepsin L inhibition on TGF- $\beta$-induced Cxcr-7 was examined by western blot analysis. At least three independent experiments were performed. ${ }^{*} \mathrm{P}<0.05,{ }^{* *} \mathrm{P}<0.01$ and ${ }^{* * * *} \mathrm{P}<0.001$ compared with control. ${ }^{\#} \mathrm{P}<0.05,{ }^{\# \#} \mathrm{P}<0.01$ and ${ }^{\# \# \#} \mathrm{P}<0.001$ compared with the TGF- $\beta$ group. TGF- $\beta$, transforming growth factor- $\beta$.

receptors, and this effect was inhibited by cathepsin L knockdown in A549 and MCF-7 cells (Fig. 2E). The enhanced ability of tumor invasion and migration by inhibiting cathepsin $\mathrm{L}$ in the TGF- $\beta$-treated cells suggest an important role of cathepsin L in the progression of tumor invasion and migration.

Cathepsin L knockdown blocks TGF- $\beta$-induced EMT. Suppression of cathepsin $\mathrm{L}$ in breast cancer and several other types of carcinomas has been reported to correlate with tumor invasion and migration. Nevertheless, the mechanisms by which cathepsin $\mathrm{L}$ weakens the ability of cell invasion and migration remain to be clarified. To examine whether changes of the cathepsin L protein level are associated with TGF- $\beta$-mediated EMT, we knocked down the expression of cathepsin $\mathrm{L}$ in the two cancer lines treated with TGF- $\beta$. At the end of $24 \mathrm{~h}$, compared with the untreated cells, we found that EMT was induced in TGF- $\beta$-treated cells. Furthermore, when cathepsin $\mathrm{L}$ was switched from a high expression to a low expression state, we assessed the modulation of $\mathrm{E}$ - and N-cadherin protein, two hallmarks of EMT, using IF and western blot 

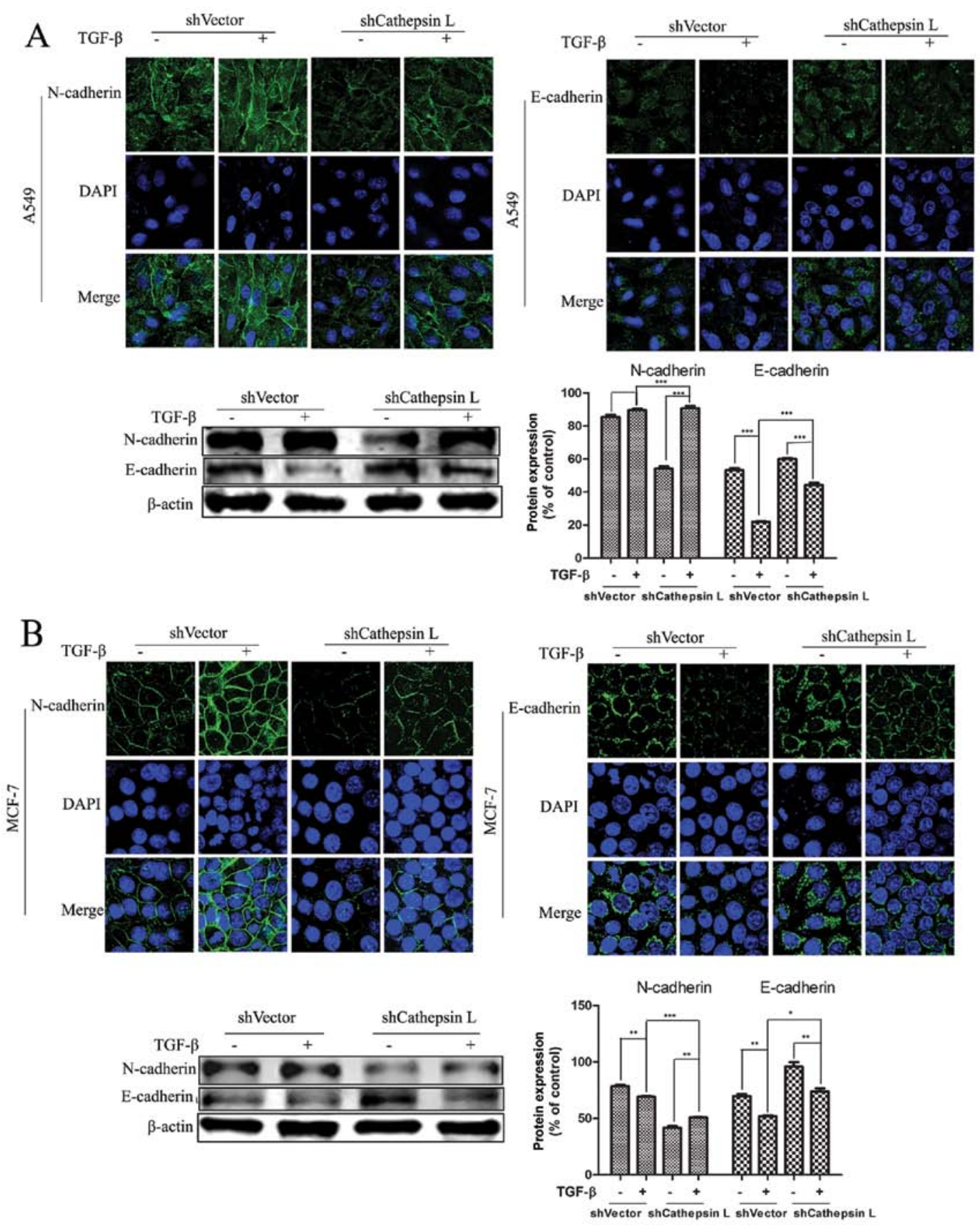

Figure 3. Stable transfection of shCathepsin L attenuates TGF- $\beta$-induced EMT. (A) A549 cells were treated for $24 \mathrm{~h}$ with TGF- $\beta$ ( $5 \mathrm{ng} / \mathrm{ml})$. N- and E-cadherin were subjected to immunofluorescent microscopy or were assessed by western blot analysis. Images were captured using a 40x objective lens. (B) MCF-7 cells were treated for $24 \mathrm{~h}$ with TGF- $\beta(10 \mathrm{ng} / \mathrm{ml})$. N- and E-cadherin were subjected to immunofluorescent microscopy or were assessed by western blot analysis. Images were captured using a 40x objective lens. At least three independent experiments were performed. ${ }^{*} \mathrm{P}<0.05,{ }^{* * *} \mathrm{P}<0.01$ and ${ }^{* * * *} \mathrm{P}<0.001$ compared with control. ${ }^{\#} \mathrm{P}<0.05,{ }^{\# \#} \mathrm{P}<0.01$ and ${ }^{\# \# \#} \mathrm{P}<0.001$ compared with the TGF- $\beta$ group. TGF- $\beta$, transforming growth factor $-\beta$; IF, immunofluorescence.

analysis. Notably, in A549 cells, the knockdown of cathepsin $\mathrm{L}$ result in blunting of TGF- $\beta$-mediated EMT, as demonstrated by a decrease in the amount of $\mathrm{N}$-cadherin and an increase of E-cadherin. By contrast, these alterations were not observed in the untransfected cells with shCathepsin L (Fig. 3). The same experiments were performed in the MCF-7 cell line and the results were consistent with results for the A549 cells. These results suggested that blocking of EMT by inhibiting cathepsin $\mathrm{L}$ indicates a regulatory role for cathepsin $\mathrm{L}$ in mediating the TGF- $\beta$-induced EMT and cell migration.
Cathepsin L regulates the expression of molecules involved in EMT. Overexpression of cathepsin $\mathrm{L}$ in lung cancer and other types of carcinoma has been reported to be correlated with tumorigenesis and development. Nevertheless, the mechanism by which cathepsin $\mathrm{L}$ promotes the invasive and migratory ability of tumor cells remains to be clarified. Findings of recent studies showed that Snail is an essential regulator of EMT and mediates invasiveness as well as metastasis in various malignant tumors $(2,39,40)$. To verify whether there was an association of the function of Snail and activity of 

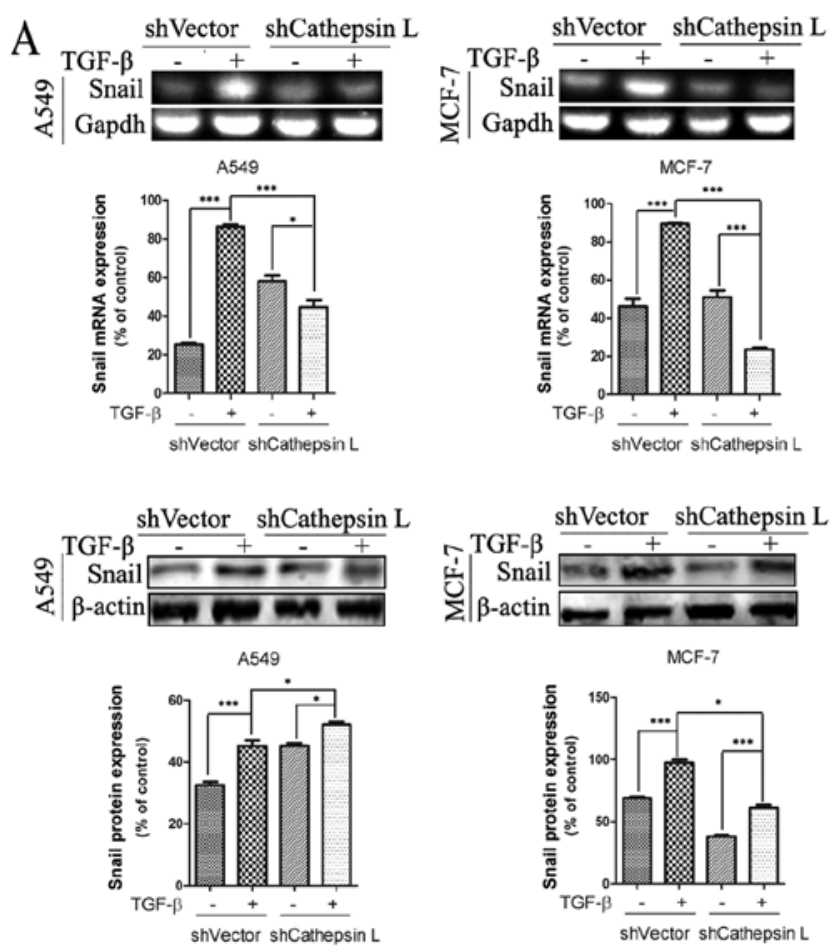
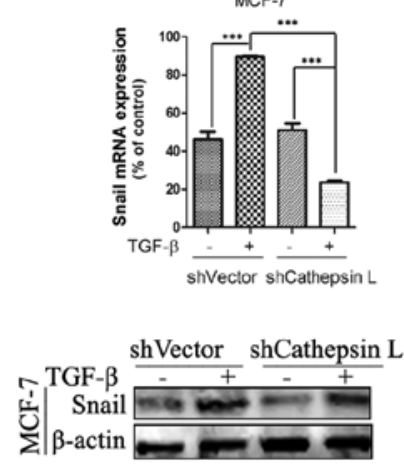

MCF-7

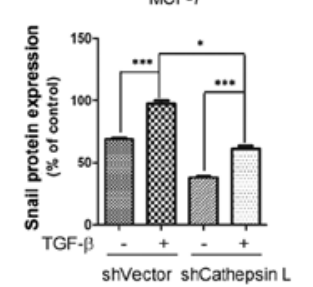

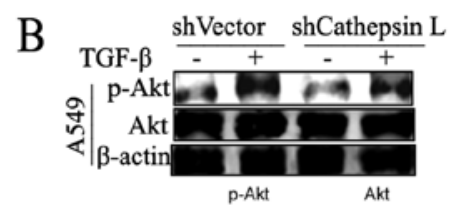
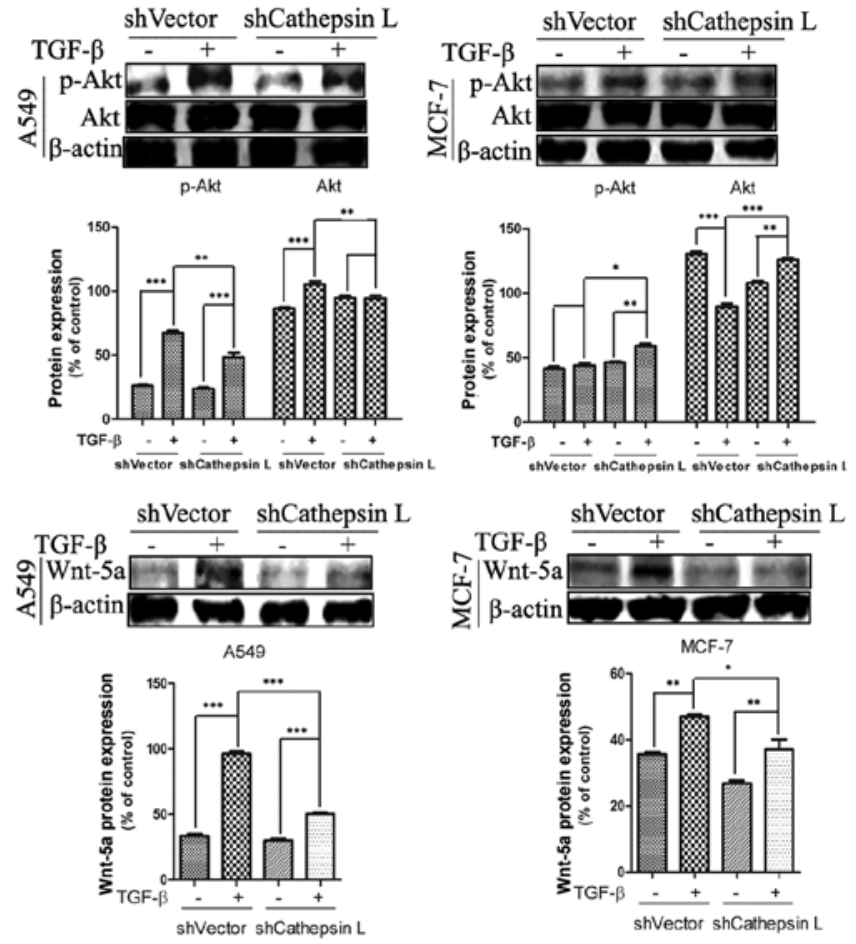

Figure 4. Effect of stable transfection of shCathepsin L on Snail, p-Akt and Wnt-5a expression levels. (A), A549 and MCF-7 cells were treated with TGF- $\beta$. The level of Snail was analysed by RT-PCR and western blot analysis. (B), A549 and MCF-7 cells were treated with TGF- $\beta$. The levels of Akt and Wnt-5a were analysed by western blot analysis. At least three independent experiments were performed. ${ }^{*} \mathrm{P}<0.05,{ }^{* *} \mathrm{P}<0.01$ and ${ }^{* * * *} \mathrm{P}<0.001$ compared with control. ${ }^{\#} \mathrm{P}<0.05$, ${ }^{\# \#} \mathrm{P}<0.01$ and ${ }^{\# \# \#} \mathrm{P}<0.001$ compared with the TGF- $\beta$ group. TGF- $\beta$, transforming growth factor- $\beta$.

cathepsin LA549 and MCF-7 cells lines were used in which shCathepsin L was or was not introduced. We observed that TGF- $\beta$ treatment induced an increase in the mRNA and protein levels of Snail in A549 and MCF-7 cells. By contrast, stable transfection of shCathepsin L suppressed TGF- $\beta$-induced Snail gene expression (Fig. 4A). These results support the hypothesis that cathepsin $\mathrm{L}$ regulates the expression of Snail and is involved in the regulation of TGF- $\beta$-induced EMT in A549 and MCF-7 cells. In addition, Snail was induced by other signaling pathways such as Wnt and phosphatidylinositol 3-kinase (PI3K)-AKT pathways to induce EMT (41-43). As shown in Fig. 4B, transfection of cathepsin $\mathrm{L}$ abrogated the TGF- $\beta$-induced increase of $\mathrm{p}$-Akt and Wnt-5a protein levels in A549 and MCF-7 cells. These data indicate that cathepsin L, an EMT regulator functions via a mechanism involving the effect of Snail expression, Wnt and PI3K-AKT pathways.

Cathepsin L contributes to regulating EMT in vivo. Based on the in vitro experiment results, the effect of cathepsin $\mathrm{L}$ on TGF- $\beta$-mediated EMT was more significant in A549 than MCF-7 cells. Thus, to further validate the role of cathepsin $\mathrm{L}$ in EMT, two clones of A549 derivative cells with cathepsin L knockdown (shCathepsin L) and a non-target control (shVector) were injected into athymic nude mice subcutaneously to generate the mouse model.

After 42 days, the tumor size was measured and the tumor volume in A549 cells with cathepsin L knockdown was found to have significant shrinkage compared with the non-target control (Fig. 5A), suggesting the ability of cell proliferation was blunted. Downregulation of E-cadherin, with upregulation of N-cadherin is known to be a key indicator of the EMT process (44). Immunohistochemical staining and western blot analysis were used to analyze the protein expression of the epithelial markers E-cadherin and the mesenchymal markers $\mathrm{N}$-cadherin in tumor tissue. As shown in Fig. 5B, an increased level of E-cadherin and a decreased level of $\mathrm{N}$-cadherin were observed in cathepsin L knockdown (shCathepsin L) tumor tissue, compared with the non-target control tumor tissue.

These data, along with the results of the in vitro analysis, proved that cathepsin L knockdown suppresses tumor invasion and migration by inhibiting EMT, confirming the role of cathepsin L in further regulating EMT.

\section{Discussion}

In the present study, we report a previously unrecognized role of cathepsin L in regulating EMT and provide a possible mechanistic role for how cathepsin L inhibition contributes to the decreased cell invasion and migration. In the present study, two types of epithelial tumor cells, human MCF-7 breast and human A549 lung carcinoma cell lines were selected as the model system. Our results show that the effect of cathepsin $\mathrm{L}$ knockdown on the suppression of TGF- $\beta$-induced cell migration and invasion was associated with its regulation of EMT. Its mechanism may be explained by suppression of the EMT-inducing molecules, such as Snail, which is associated with the PI3K-AKT and Wnt signaling pathways. The findings concerning the function of cathepsin $\mathrm{L}$ in TGF- $\beta$-induced EMT provide new evidence on the regulation of cancer invasion and migration. 

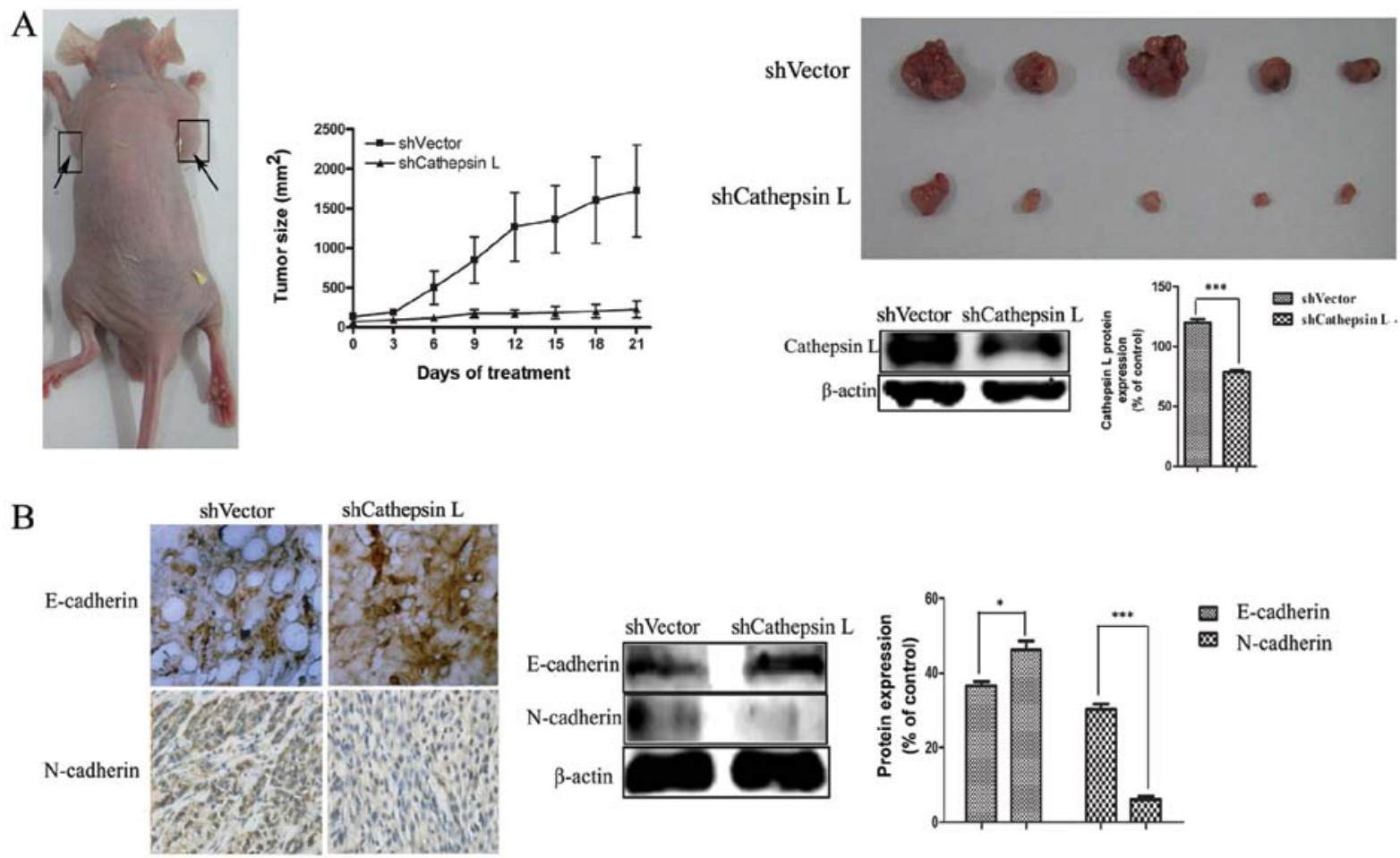

Figure 5. The effect of stable transfection of shCathepsin L on EMT in vivo. A549 and A549-Cathepsin L shRNA cells (5x10 ${ }^{7}$ cells/0.1 ml of medium/mouse) were injected subcutaneously to generate the mouse model. (A) The general appearance, tumor size, and level of cathepsin L in the mice 42 days after inoculation of A549 and A549-Cathepsin L shRNA cells. (B) The expression of N- and E-cadherin was compared between the two groups by immunohistochemical staining and western blot analysis. Images were captured using a 20x objective lens. At least three independent experiments were performed. ${ }^{*} \mathrm{P}<0.05,{ }^{* *} \mathrm{P}<0.01$ and ${ }^{* * *} \mathrm{P}<0.001$ compared with control. ${ }^{\#} \mathrm{P}<0.05,{ }^{\# \#} \mathrm{P}<0.01$ and ${ }^{\# \# "} \mathrm{P}<0.001$ compared with the TGF- $\beta$ group. EMT, epithelial-mesenchymal transition; TGF- $\beta$, transforming growth factor- $\beta$.

Accumulating evidence reveals that TGF- $\beta$ is often used as the inducer of EMT to increase tumor invasion and migration. A549 is K-Ras mutation, whereas MCF-7 is not and it has been shown that active Ras is required for TGF- $\beta$-induced EMT (45). Thus, we treated A549 and MCF-7 cells with different concentrations of TGF- $\beta$. The results showed that the cell morphology changes of EMT occurred $\sim 24 \mathrm{~h}$ after TGF- $\beta$ treatment (Fig. 1A), while the expression of cathepsin $\mathrm{L}$ was increased (Fig. 1B). Therefore, the expression of cathepsin $\mathrm{L}$ is associated with EMT.

Cathepsin L, which is less well studied than cathepsin B, has been found to be associated with tumor invasion and migration (23). In various tumor types, such as melanoma, lung and breast cancer $(21,31,46)$, the functional significance of cathepsin $\mathrm{L}$ in invasion is appreciated. Our results show that cathepsin L may induce the morphological changes of A549 and MCF-7 cells and enhance the migration and invasion in the two cell lines. Of note, the migration and invasion of cells was positively correlated with the expression of cathepsin L. Thus, when the invasion ability peaked, the expression of cathepsin $\mathrm{L}$ also peaked. To determine whether the migration and invasion change of A549 and MCF-7 cells corresponded with the expression of cathepsin L, we suppressed the expression of cathepsin $\mathrm{L}$ via transfection with shCathepsin $\mathrm{L}$. The results show that cathepsin $\mathrm{L}$ knockdown strongly suppressed TGF- $\beta$-mediated cell migration, invasion and actin remodeling associated with cell motility. Moreover, cathepsin L knockdown inhibited the expression of Cxcr-7, which is important in tumor invasion and migration.
The expression of cathepsin $\mathrm{L}$ is known to regulate invasion and migration in various types of cells. However, the underlying mechanisms remain to be clarified. Krueger et al demonstrated that cathepsin $\mathrm{L}$ activates cathepsin $\mathrm{B}$, triggering a chain reaction and inducing the activation of uPA, which synergistically promotes tumor cell invasion and migration (47). Cathepsin L stimulated the release of some growth factors by degrading the extracellular matrix in order to further promote tumor invasion and migration (33). Furthermore, Gocheva et al suggested that cathepsin $\mathrm{L}$ decreases the cell-cell adhesion to enhance tumor invasion and migration by its direct cleavage of E-cadherin (48), a marker protein of EMT. Considering the existing results obtained in our laboratory, it is likely that cathepsin L suppresses cancer invasion and migration by inhibiting the EMT process. To detect whether the effect of cathepsin $\mathrm{L}$ on cell migration and invasion was associated with EMT, we performed IF and western blot analysis to assess expression of E- and N-cadherin, which are EMT-associated proteins. Inactivation of E-cadherin is one of the key events in EMT. On the other hand, activation of an inappropriate cadherin, such as N-cadherin, may be a subsequent event and promote tumor invasion and migration (37). Our results show that high $\mathrm{N}$-cadherin but low E-cadherin levels were observed after TGF- $\beta$ treatment, suggesting that TGF- $\beta$ induced EMT in A549 and MCF-7 cells. However, cathepsin L knockdown inhibited EMT in response to TGF- $\beta$ and restored epithelial phenotypes in tumor cells (Fig. 3), suggesting that cathepsin L may function as an EMT regulator in various types of epithelial tumor cells. 
Regulation of EMT is a complex process involving multiple genes and the pathways in embryonic development and in normal and transformed cell lines, such as extracellular signal-regulated protein kinases (ERKs), PI3K/Akt, Smads and Wnts, and is significantly associated with the aggressiveness and metastatic potential of cancer $(49,50)$. In addition, it is more likely that the majority of signaling pathways known to trigger EMT converge at the induction of the E-cadherin repressors (49). Loss of E-cadherin expression is considered an important step in the progression of tumor metastasis, and a fundamental event in EMT (49).

Snail, a member of the Snail family of zinc finger transcription factors, is a suppressor of the transcription of shotgun (an E-cadherin homologue). It is a widely used inducer of EMT in various types of human cancer of epithelial origin, is known as a repressor of $E$-cadherin gene and is important in TGF- $\beta$-induced EMT $(51,52)$. Snail was able to regulate the changes in gene expression patterns that underlie EMT $(6,51)$.

Our results show that cathepsin L knockdown abrogated TGF- $\beta$-induced expression of Snail at the mRNA and protein levels (Fig. 4A). This result suggests that Snail may be significantly involved in the regulation of EMT by cathepsin L. It also shows that PI3K/Akt and Wnt signaling pathways affect the expression of epithelial markers to regulate the EMT process $(49,50)$. PI3K-Akt signaling pathways participate in regulating cell proliferation, differentiation, survival and migration. In recent years, we found that the activity of this pathway abnormality may cause malignant cell transformation and is associated with invasion and migration of tumor cell. Wnt signaling has an important role in embryonic development, and its deregulation is closely associated with the occurrence of a number of malignant tumors, including breast and colon cancer. Lee $e t$ al suggested that it induces Snail-dependent EMT, which is responsible for tumor invasion and migration (53). In addition, WNT-5A is a transcriptional target of TGF- $\beta$ whose expression was induced by TGF- $\beta$ (54).

In the present study, we observed the enhanced activation of PI3K/Akt and Wnt signaling pathways in TGF- $\beta$-treated cells. However, cathepsin L knockdown suppressed the enhanced expression of p-Akt and Wnt-5a. Therefore, we considered that the mechanism of how cathepsin L knockdown regulates EMT may be explained by the suppression of Snail genes, and the PI3K-AKT and Wnt signaling pathways (Fig. 4B).

In the process of tumor progression, cells proliferate and differentiate abnormally, with the proliferative and invasive ability becoming stronger via the EMT. To confirm the role of cathepsin L in regulating EMT, we determined whether cathepsin L knockdown in vivo may prevent EMT. In this experiment, the tumor growth rate and expression level of EMT-associated proteins were measured to detect EMT rangeability. The results demonstrate the in vitro findings that, cathepsin L regulates EMT in vivo. Tumor volume in cathepsin L knockdown cells had significant shrinkage compared with the non-target control. An increase in E-cadherin and a decreased in the $\mathrm{N}$-cadherin levels were observed in cathepsin L knockdown (shCathepsin L) tumor tissue (Fig. 5) were observed, providing further experimental evidence supporting the role of cathepsin L in the regulation of EMT. These results show that cathepsin L knockdown is efficacious for EMT inhibition in tumor cells.
In summary, the present study has identified cathepsin $\mathrm{L}$ as a novel regulator of EMT and its involvement in the modulation of tumor cell invasion and migration. Additonally, inhibition of cathepsin L suppresses the increased cell invasion and migration induced by TGF- $\beta$. These findings may expand current knowledge of EMT networks and shed light on how EMT is regulated in epithelial tumor cells. Thus, cathepsin L may be examined as a novel therapeutic target for the prevention of tumor metastasis by regulating EMT.

\section{References}

1. Thiery JP and Sleeman JP: Complex networks orchestrate epithelial-mesenchymal transitions. Nat Rev Mol Cell Biol 7: 131-142, 2006.

2. Fan F, Samuel S, Evans KW, Lu J, Xia L, Zhou Y, Sceusi E, Tozzi F, Ye XC, Mani SA and Ellis LM: Overexpression of snail induces epithelial-mesenchymal transition and a cancer stem cell-like phenotype in human colorectal cancer cells. Cancer Med 1: 5-16, 2012.

3. Shi J, Wang DM, Wang CM, Hu Y, Liu AH, Zhang YL, Sun B and Song JG: Insulin receptor substrate-1 suppresses transforming growth factor- $\beta 1$-mediated epithelial-mesenchymal transition. Cancer Res 69: 7180-7187, 2009.

4. Voulgari A and Pintzas A: Epithelial-mesenchymal transition in cancer metastasis: mechanisms, markers and strategies to overcome drug resistance in the clinic. Biochim Biophys Acta 1796: 75-90, 2009.

5. Hugo H, Ackland ML, Blick T, Lawrence MG, Clements JA, Williams ED and Thompson EW: Epithelial - mesenchymal and mesenchymal - epithelial transitions in carcinoma progression. $\mathbf{J}$ Cell Physiol 213: 374-383, 2007.

6. Saito RA, Watabe T, Horiguchi K, Kohyama T, Saitoh M, Nagase $\mathrm{T}$ and Miyazono K: Thyroid transcription factor-1 inhibits transforming growth factor- $\beta$-mediated epithelial-to-mesenchymal transition in lung adenocarcinoma cells. Cancer Res 69: 2783-2791, 2009.

7. Lim S, Becker A, Zimmer A, Lu J, Buettner R and Kirfel J: SNAI1-mediated epithelial-mesenchymal transition confers chemoresistance and cellular plasticity by regulating genes involved in cell death and stem cell maintenance. PLoS One 8: e66558, 2013

8. Pardali K and Moustakas A: Actions of TGF-beta as tumor suppressor and pro-metastatic factor in human cancer. Biochim Biophys Acta 1775: 21-62, 2007.

9. Gupta M, Korol A and West-Mays JA: Nuclear translocation of myocardin-related transcription factor-A during transforming growth factor beta-induced epithelial to mesenchymal transition of lens epithelial cells. Mol Vis 19: 1017-1028, 2013.

10. Naber HP, Drabsch Y, Snaar-Jagalska BE, et al: Snail and Slug, key regulators of TGF- $\beta$-induced EMT, are sufficient for the induction of single-cell invasion. Biochem Biophys Res Commun 435: 58-63, 2013.

11. Levicar N, Nuttall RK and Lah TT: Proteases in brain tumor progression. Acta Neurochir 145 (Wien): 825-838, 2003.

12. Yong VW: Metalloproteinases: mediators of pathology and regeneration in the CNS. Nat Rev Neurosci 6: 931-944, 2005.

13. Harada T, Arii S, Mise M, et al: Membrane-type matrix metalloproteinase-1(MT 1-MMP) gene is overexpressed in highly invasive hepatocellular carcinomas. J Hepatol 28: 231-239, 1998.

14. Wang JR, Li XH, Gao XJ, et al: Expression of MMP-13 is associated with invasion and metastasis of papillary thyroid carcinoma. Eur Rev Med Pharmacol Sci 17: 427-435, 2013.

15. Brix K, Dunkhorst A, Mayer K and Jordan S: Cysteine cathepsins: cellular roadmap to different functions. Biochimie 90: 194-207, 2008.

16. Bylaite M, Moussali H, Marciukaitiene I, et al: Expression of cathepsin $\mathrm{L}$ and its inhibitor hurpin in inflammatory and neoplastic skin diseases. Exp Dermatol 15: 110-118, 2006.

17. Stahl S, Reinders Y, Asan E, et al: Proteomic analysis of cathepsin B- and L-deficient mouse brain lysosomes. Biochimica Biophysica Acta 1774: 1237-1246, 2007.

18. Skrzydlewska E, Sulkowska M, Koda M and Sulkowski S: Proteolytic-antiproteolytic balance and its regulation in carcinogenesis. World J Gastroenterol 11: 1251-1266, 2005. 
19. Navab R, Pedraza C, Fallavollita L, et al: Loss of responsiveness to IGF-I in cells with reduced cathepsin L expression levels. Oncogene 27: 4973-4985, 2008.

20. Pucer A, Castino R, Mirković B, et al: Differential role of cathepsins $\mathrm{B}$ and $\mathrm{L}$ in autophagy-associated cell death induced by arsenic trioxide in U87 human glioblastoma cells. Biol Chem 391: 519-531, 2010

21. Zajc I, Hreljac I and Lah T: Cathepsin L affects apoptosis of glioblastoma cells: a potential implication in the design of cancer therapeutics. Anticancer Res 26: 3357-3364, 2006.

22. Strojnik T, Kos J, Zidanik B, et al: Cathepsin B immunohistochemical staining in tumor and endothelial cells is a new prognostic factor for survival in patients with brain tumors. Clin Cancer Res 5: 559-567, 1999.

23. Kirschke H, Eerola R, Hopsu-Havu VK, et al: Antisense RNA inhibition of cathepsin $\mathrm{L}$ expression reduces tumorigenicity of malignant cells. Eur J Cancer 36: 787-795, 2000.

24. Gocheva V and Joyce JA: Cysteine cathepsins and the cutting edge of cancer invasion. Cell Cycle 6: 60-64, 2007.

25. Jedeszko $C$ and Sloane BF: Cysteine cathepsins in human cancer. Biol Chem 385: 1017-1027, 2004.

26. Mohamed MM and Sloane BF: Cysteine cathepsins: multifunctional enzymes in cancer. Nat Rev Cancer 6: 764-775, 2006.

27. Strojnik T, Kavalar R, Trinkaus M and Lah TT: Cathepsin L in glioma progression: comparison with cathepsin B. Cancer Detect Prev 29: 448-455, 2005

28. Chambers AF, Colella R, Denhardt DT and Wilson SM: Increased expression of cathepsins $\mathrm{L}$ and $\mathrm{B}$ and decreased activity of their inhibitors in metastatic, ras-transformed NIH 3T3 cells. Mo Carcinog 5: 238-245, 1992.

29. Frade R, Rodrigues-Lima F, Huang S, et al: Procathepsin-L, a proteinase that cleaves human $\mathrm{C} 3$ (the third component of complement), confers high tumorigenic and metastatic properties to human melanoma cells. Cancer Res 58: 2733-2736, 1998.

30. Yang Z and Cox JL: Cathepsin L increases invasion and migration of B16 melanoma. Cancer Cell Int 7: 8, 2007.

31. Rousselet N, Mills L, Jean D, et al: Inhibition of tumorigenicity and metastasis of human melanoma cells by anti-cathepsin L single chain variable fragment. Cancer Res 64: 146-151, 2004.

32. Wang SM, Li L, Zhang W, et al: Relationship between cathepsin L and invasion and metastasis of ovarian carcinoma cells. Zhonghua Fu Chan Ke Za Zhi 45: 598-602, 2010 (In Chinese).

33. Lah TT, Durán Alonso MB and Van Noorden CJ: Antiprotease therapy in cancer: hot or not? Expert Opin Biol Ther 6: 257-279, 2006.

34. Goulet B, Sansregret L, Leduy L, et al: Increased expression and activity of nuclear cathepsin $L$ in cancer cells suggests a novel mechanism of cell transformation. Mol Cancer Res 5: 899-907, 2007.

35. Krueger S, Kellner U, Buehling F, et al: Cathepsin L antisense oligonucleotides in a human osteosarcoma cell line: effects on the invasive phenotype. Cancer Gene Ther 8: 522-528, 2001.

36. Matarrese P, Ascione B, Ciarlo L, et al: Cathepsin B inhibition interferes with metastatic potential of human melanoma: an in vitro and in vivo study. Mol Cancer 9: 207, 2010.

37. Shintani Y, Okimura A, Sato K, et al: Epithelial to mesenchymal transition is a determinant of sensitivity to chemoradiotherapy in non-small cell lung cancer. Ann Thorac Surg 92: 1794-1804, 2011.
38. Hao M, Zheng J, Hou K, et al: Role of chemokine receptor CXCR7 in bladder cancer progression. Biochem Pharmacol 84: 204-214, 2012

39. Bi WR, Jin CX, Xu GT and Yang CQ: Bone morphogenetic protein-7 regulates Snail signaling in carbon tetrachloride-induced fibrosis in the rat liver. Exp Ther Med 4: 1022-1026, 2012.

40. Nieto MA: The Snail superfamily of zinc-finger transcription factors. Nat Rev Mol Cell Biol 3: 155-166, 2002.

41. Shin SY, Rath O, Zebisch A, et al: Functional roles of multiple feedback loops in extracellular signal-regulated kinase and Wnt signaling pathways that regulate epithelial-mesenchymal transition. Cancer Res 70: 6715-6724, 2010.

42. Eger A, Stockinger A, Park J, et al: beta-Catenin and TGFbeta signalling cooperate to maintain a mesenchymal phenotype after FosER-induced epithelial to mesenchymal transition. Oncogene 23: 2672-2680, 2004.

43. Xiao D and He J: Epithelial mesenchymal transition and lung cancer. J Thorac Dis 2: 154-159, 2010.

44. Chunhacha P, Sriuranpong V and Chanvorachote P: Epithelialmesenchymal transition mediates anoikis resistance and enhances invasion in pleural effusion-derived human lung cancer cells. Oncol Lett 5: 1043-1047, 2013.

45. Davies M, Robinson M, Smith E, et al: Induction of an epithelial to mesenchymal transition in human immortal and malignant keratinocytes by TGF-betal involves MAPK, Smad and AP-1 signalling pathways. J Cell Biochem 95: 918-931, 2005.

46. Amuthan G, Biswas G, Ananadatheerthavarada HK, et al: Mitochondrial stress-induced calcium signaling, phenotypic changes and invasive behavior in human lung carcinoma A549 cells. Oncogene 21: 7839-7849, 2002.

47. Krueger S, Kellner U, Buehling F and Roessner A: Cathepsin L antisense oligonucleotides in a humall osteosarcoma cell line: efects on the invasive phenotype. Cancer Gene Ther 8: 522-528, 2001.

48. Gocheva V, Zeng W, Ke D, et al: Distinct roles for cysteine cathepsin genes in multistage tumorigenesis. Genes Dev 20: 543-556, 2006.

49. Thiery JP, Acloque H, Huang RY and Nieto MA: Epithelialmesenchymal transitions in development and disease. Cell 139: 871-890, 2009

50. Zhang Q, Bai X, Chen $\mathrm{W}$, et al: Wnt/ $\beta$-catenin signaling enhances hypoxia-induced epithelial-mesenchymal transition in hepatocellular carcinoma via crosstalk with hif-1 $\alpha$ signaling. Carcinogenesis 34: 962-973, 2013

51. Peinado H, Olmeda D and Cano A: Snail, Zeb and bHLH factors in tumour progression: an alliance against the epithelial phenotype? Nat Rev Cancer 7: 415-428, 2007.

52. Hanahan D: Heritable formation of pancreatic beta-cell tumours in transgenic mice expressing recombinant insulin/simian virus 40 oncogenes. Nature 315: 115-122, 1985.

53. Lee SY, Jeon HM, Ju MK, et al: Wnt/Snail signaling regulates cytochrome c oxidase and glucose metabolism. Cancer Res 72: 3607-3617, 2012.

54. Kumawat K, Menzen MH, Slegtenhorst RM, Halayko AJ, Schmidt $M$ and Gosens R: TGF- $\beta$-activated kinase 1 (TAK1) signaling regulates TGF- $\beta$-induced WNT-5A expression in airway smooth muscle cells via $\mathrm{Sp} 1$ and $\beta$-catenin. PLoS One 9: e94801, 2014 